\title{
Éditorial:
}

\section{Une psychologie du vieillissement: qui en a besoin?}

Presque chaque année, à l'assemblée annuelle de l'Association canadienne de gérontologie (ACG), le petit groupe au dîner de la division de la psychologie organise un référendum informel: devons-nous demeurer distincts ou faire partie d'une plus grande division au sein de l'ACG, comme celle des sciences sociales? Malgré le fait que plusieurs intervenants autour de cette table œuvrent au sein d'équipes ou de départements multidisciplinaires, nous arrivons toujours au même résultat lors de ces discussions. La psychologie a besoin de se distinguer par l'entremise de sa propre division. Pourquoi?

Le sociologue Stephen Katz, de l'Université de Trent, affirme dans son livre The Disciplining of Old Age (1996), à la suite de Foucault, que les disciplines construisent des représentations dominantes du monde et qu'elles "déterminent les manières dont les gens quil'habitent peuvent être connus, étudiés, calculés, formés, aidés, punis et libérés» (p. 2). On pourrait soutenir que les gérontopsychologues sont doublement coupables de restreindre les connaissances, premièrement par leur décision de séparer la vieillesse du reste de la durée de vie, et deuxièmement en tournant une lentille singulièrement psychologique sur les questions qui émergent d'un examen des personnes âgées. Il est sûr qu'un coup d'œil sur les articles savants traitant de la psychologie du vieillissement révèle des choses pas mal ésotériques; par exemple, les effets de l'âge sur le mouvement saccadé des yeux, la mise en condition négative (negative priming) et la sélectivité émotionnelle. Ce matériel ne devient pas facilement nomade, c'est-à-dire qu'il ne migre pas facilement vers d'autres groupes d'âges ou d'autres disciplines. Katz a alors peut-être raison. La disciplinarité contraint ce qui peut être considéré comme des sujets d'étude valides et authentiques, et nuit à une étude ouverte et critique des hypothèses derrière ces domaines.

Néanmoins, avant d'incorporer la psychologie au reste des sciences sociales et biologiques, considérons sa contribution à notre compréhension du vieillissement. Les psychologues se sont intéressés depuis longtemps à la croissance des gens durant leur vie. Les premiers spécialistes de la croissance se sont attardés à l'enfance, une période de changement rapide et généralement progressif. Ces chercheurs ont trouvé l'approche organismique de Piaget utile pour comprendre une période de la vie où la biologie avait une incidence si claire et si puissante sur la croissance. Cependant, à mesure que de plus en plus de chercheurs tentaient d'appliquer le même raisonnement à l'âge adulte, ils ont compris les limitations d'une perspec- 
tive organismique. L'organicisme semblait les pousser vers les tenants de l'approche de la détérioration biologique: une fois que les croissances psychologique et physique sont "atteintes» au début de l'âge adulte, les deux doivent alors nécessairement péricliter en égale harmonie dans le cadre du processus de vieillissement.

Les psychologues n'étaient pas trop heureux de l'idée que tout ce qu'ils pouvaient anticiper au moment de recevoir leurs doctorats était un déclin graduel de leur fonctionnement. L'âge avancé les a laissés encore plus irrités de ce point de vue (par ex., Skinner, 1983).C'est peut-être pour cette raison que l'approche du développement au cours de la vie de Paul Baltes (par ex., 1987) a connu un attrait aussi rapide. Baltes a émis l'hypothèse que le développement se produisait dans un contexte, au cours de toute une vie, et qu'il pouvait être progressif ou régressif. Il était alors possible de percevoir le vieillissement psychologique comme fermement ancré dans un contexte social. Les chercheurs ont été encouragés à aller au-delà de l'observation que les déclins de la mémoire active sont courants dans la deuxième moitié de la vie pour comprendre que pareils déclins ne sont aucunement universels et qu'ils sont grandement influencés par l'histoire de vie personnelle et l'environnement de l'individu.

Ce point de vue aurait sûrement plu à B. F. Skinner qui, dans son article sur son propre vieillissement, paru en 1983 dans American Psychologist, a incité les chercheurs à rejeter la métaphore horticole sur le vieillissement - c'est-à-dire pourrir et se décomposer - pour réaliser que «beaucoup de ce qui semble être le déploiement d'un potentiel intérieur [avec croissance] est le produit d'un environnement épanouissant: l'univers d'une personne se développe» (p. 239). D'après cette façon de penser, Skinner a laissé entendre que les praticiens devraient consacrer plus de temps à réfléchir au sujet d' "environnements prosthétiques," c'est-à-dire d'environnements qui procurent aux personnes âgées une vaste gamme d'expériences et de stimulation cognitive. Ces environnements stimulants atténueraient le changement des conditions de renforcement qui produisent l'ennui et le manque d'intérêt durant la vieillesse. Si la vie ne fournit plus assez de motivations - à cause de la perte d'emploi, du manque d'attention de la part de ses enfants, ou des réseaux sociaux restreints - les déclins dans le fonctionnement sont presque inévitables.

Les points de vue de Skinner étaient essentiellement mécaniques et plusieurs psychologues sont plutôt embarrassés s'ils doivent avouer qu'ils les admirent. Néanmoins, des fils de sa théorie semblent profondément tissés dans la réflexion actuelle sur la psychologie du vieillissement. Une théorie prometteuse découlant du cadre de croissance durant toute la vie, de Baltes - l'optimisation sélective avec compensation - met une emphase particulière sur les effets micro et macro de l'environnement sur la croissance (Baltes, 1997). Le premier élément de ce cadre, la sélection, fait référence aux choix que font des individus - consciemment ou non - pour se concentrer sur des domaines de croissance personnelle dans lesquels ils 
sont susceptibles de réussir (par ex., jouer aux échecs plutôt qu'au tennis). Le deuxième élément, l'optimisation, fait référence au maintien d'habiletés ou compétences qui ont déjà été acquises (par ex., la répétition quotidienne des concertos pour violoncelle de Bach appris dans sa jeunesse). Le troisième élément, la compensation, fait référence aux efforts que fait un individu pour maintenir ses compétences quand il y a eu des pertes, que des objectifs sont devenus incompatibles ou que de nouvelles contraintes ont été imposées (par ex., rechercher une nouvelle relation d'intimité après être devenu veuf ou veuve). Cette méthode reconnaît que des pertes ou des restrictions peuvent résulter du vieillissement, mais aussi que la personne âgée peut s'adapter pour demeurer le plus fonctionnelle possible.

Comme je l'ai déjà signalé ailleurs (Pratt \& Norris, sous presse), l'optimisation sélective avec compensation fournit un modèle explicite pour conceptualiser «le vieillissement réussi". La façon dont vieillissent avec succès les aîné(e)s est en voie de devenir une préoccupation importante pour les praticiens en psychologie et les chercheurs. C'est peut-être en partie à cause de nos propres expériences et espoirs pour le futur que nous semblons déterminés à découvrir les "secrets" du bon vieillissement. À la veille de l'Année internationale des aînés, ceci semble une mission appropriée. Nous sommes entourés par des prototypes possibles de personnes ayant réussi leur vieillissement. Par exemple, le secrétaire-général de l'ONU, Kofi Annan, âgé de 60 ans, et l'astronaute qui a fait une renaissance, John Glenn, acclamé comme «un pionnier de la longévité» avant son voyage dans l'espace en octobre dernier (Reuters, 1998). Le comité non gouvernemental onusien sur le vieillissement est allé plus loin en qualifiant Glenn de «non seulement un exemple pour nous tous en âge avancé, [mais] un exemple pour toutes les générations».

Au moins une personne de la génération $X$ (Foust, 1998) a contesté la valeur de mettre en évidence les histoires de gens comme John Glenn. Il signale que le véritable travail de recherche sur le vieillissement se perd dans l'éclat d'un événement médiatique et dans la nostalgie des personnes qui se rappellent le lancement de Mercury en 1962. Cette observation avertit les gérontologues d'examiner de près notre travail et nos traditions, alors que nous tentons de comprendre les nouveaux développements. Il y a quelques années, mon collègue Michael Pratt et moi (Pratt \& Norris, 1994) avions tenté de faire cela au moyen d'une analyse de la recherche sur la psychologie du vieillissement. Nous avions découvert que la recherche existante dérivait de l'une ou l'autre des quatre perspectives suivantes: la psychométrie, le traitement de l'information, le développement ou la sociopsychologie. Nous avions indiqué que le choix de perspective était très influencé par le phénomène à l'étude et qu'aucune perspective ne pouvait être envisagée comme étant "la meilleure» ou même la plus prometteuse dans un sens large et programmatique. Nous avions toutefois souligné l'importance d'utiliser un certain cadre théorique qui nous conduirait 
au-delà d'une analyse descriptive des phénomènes reliés au vieillissement vers une analyse explicative.

Ce numéro de la Revue canadienne du vieillissement réunit le meilleur de l'utilisation de ces approches traditionnelles à la psychologie du vieillissement avec une sérieuse tentative de découvrir le sens du vieillissement réussi dans une variété de contextes. Le document de Bouchard et de ses collègues intitule «Psychometric Properties of the French Version of the State-Trait Anxiety Inventory (form Y) Adapted for Older Adults" et l'article de Laprise et Vézina, «Diagnostic Performance of the Geriatric Depression Scale and the Beck Depression Inventory with Nursing Home Residents," s'inscrivent dans la tradition psychométrique en psychologie. Ces exposés, avec leur utilisation de tests normalisés et de conditions hautement contrôlées, nous permettent de comprendre les différences individuelles dans l'expérience du vieillissement. En plus d'améliorer notre capacité de mesurer des construits psychologiques importants comme l'anxiété et la dépression, ils nous incitent à examiner nos définitions de ces construits dans le contexte de l'âge avancé et dans celui de deux langues et de deux cultures.

L'article de Landreville et Guérette, «Psychometric Properties of a Modified Version of the Treatment Evaluation Inventory for Assessing the Acceptability of Treatments for Geriatric Depression» puise également dans la tradition psychométrique, mais il est aussi très influencé par le travail en psychologie sociale sur les perceptions. L'étude de O'Rourke et Wenaus, "Marital Aggrandizement as a Mediator of Burden Among Spouses of Suspected Dementia Patients," tisse des fils de plusieurs secteurs de la psychologie sociale - l'adaptation, les échanges sociaux et la reconstitution de souvenirs - pour explorer un médiateur important de fardeau chez les femmes, soit celui de la tendance à idéaliser le partenaire et la relation. La recherche de Fox et Gooding, «Physical Mobility and Social Integration: Their Relationship to the Well-Being of Older Canadians," adopte une position sociopsychologique dans sa considération de l'importance des liens sociaux. Ces trois articles démontrent l'utilité de l'éclectisme méthodologique et théorique de la psychologie sociale.

L'article de Patrick et Howell, "Mental Status and Self-Medication Ability in the Hospitalized Elderly," s'inspire du travail réalisé dans le traitement de l'information, pour étudier l'impact des déficits cognitifs sur la capacité de maximiser le fonctionnement dans une activité importante du quotidien. Cette étude est un important rappel que le succès en âge avancé n'est pas limité aux John Glenn de ce monde. Même des aînés frêles sur le plan cognitif peuvent conserver des compétences qui augmentent l'estime de soi et facilitent l'optimisation dans d'autres secteurs de leur vie.

Finalement, l'exposé de Doucet et de ses collègues, "Thèmes d'inquiétudes et tendance à s'inquiéter chez les aînés," puise dans plusieurs traditions: celles du traitement de l'information, de la psychologie sociale et de la croissance. Une des conclusions les plus significatives de cette étude 
a été celle de définir les personnes très âgées comme un groupe qui mérite d'être étudié plus à fond. Comme Baltes (1997) a également signalé, «Les personnes les plus âgées ne sont pas des individus qui se maintiennent en vie parce que leur état fonctionnel demeure au même niveau que celui des moins âgés» (p. 376). Leur succès à la vieillisse peut être à la fois une source d'inspiration pour les autres et peut représenter un stade unique du développement de la personne.

La recherche sur le vieillissement se trouve elle-même à un stade de développement délicat. Comme l'a souligné Katz (1996), «la vieillesse est coincée entre les efforts de la discipliner, de la calculer et de la gérer, et les forces qui l'indisciplinent, la diversifient et la fragmentents (p. 139). J'affirmerais par contre, de mon point de vue de spécialiste de l'étude du cycle de vie, que cette dialectique est une caractéristique inhérente à la croissance de notre compréhension. Et elle n'appartient pas exclusivement aux psychologues. Les gérontologues, de quelque allégeance disciplinaire que ce soit, peuvent faire avancer l'étude du vieillissement par des connaissances spécialisées, des questionnements singuliers, et la motivation de comprendre ce que signifie bien vieillir. Le sens de l'identité bien défini et fort, comme le savent les psychologues, est important pour un fonctionnement sain. Aussi, nous pouvons tous demeurer ouverts aux influences de chacun qui bouleversent nos compréhensions durement acquises et qui nous obligent à reconceptualiser notre questionnement et nous-mêmes. L'exposition à diverses relations sociales, attitudes et croyances - là encore comme le savent les psychologues - favorisent la complexité cognitive. Donc, à mes collègues qui se demandent s'ils devraient garder leurs frontières disciplinaires intactes, je dirais qu'il y a du mérite à faire cela, mais seulement si vous franchissez ces frontières occasionnellement pour le dîner.

\section{Références}

Baltes, P.B. (1987). Theoretical propositions of life-span developmental psychology: On the dynamics between growth and decline. Developmental Psychology, 23, $611-626$.

Baltes, P.B. (1997). On the incomplete architecture of human ontogeny: Selection, optimization, and compensation as foundations of developmental theory. American Psychologist, 52, 366-380.

Foust, J. (1998, November 1). Glenn again, for the very first time: A Gen-X perspective on STS-95. [On-line]. Voir: www.spaceviews.com/features/ glenn/glenn-genx.html.

Katz, S. (1996). Disciplining old age: The formation of gerontological knowledge. Charlottesville \& London: University Press of Virginia.

Pratt, M.A., \& Norris, J.E. (sous presse). Moral Development in Maturity: Lifespan Perspectives on the Processes of Successful Aging. Dans T. Hess \& F. Blanchard-Fields, Social Cognition and Aging. New York: Academic Press.

Pratt, M.A., \& Norris, J.E. (1994). The social psychology of aging: A cognitive perspective. Oxford: Blackwell. 
Reuters. (1998, October 30). U.N. lauds Glenn as "Pioneer for longevity". [On-line]. Voir: www.cnn.com/TECH/space/9810/30/un.glen.reut.

Skinner, B.F. (1983). Intellectual self-management in old age. American Psychologist, 38(3), 239-244.

Joan E. Norris 